\title{
BRIÓFITAS DA SERRA DE ITABAIANA, SERGIPE, BRASIL
}

Olga Yano'

Recebido em 08.07.93. Aceito em 23.01.94.

RESUMO - (Briófitas da Serra de Itabaiana, Sergipe, Brasil). Na Serra de Itabaiana, foram encontradas 15 espécies de musgos pertencentes a 14 gêneros de 11 famílias e 14 de hepáticas de oito gêneros de quatro famílias. Rectolejeunea pililoba (Spruce) Schust. está sendo referida pela primeira vez para o Brasil. Destas espécies, 26 estão sendo referidas pela primeira vez para o estado.

Palavras-chave: Hepaticopsida, Bryopsida, Itabaiana.

ABSTRACT - (The bryophytes from the Serra de Itabaiana, Sergipe, Brazil). Fifteen species of mosses and fourteen species of liverworts are reported from Serra de Itabaiana, Sergipe, Brazil. The mosses are distributed among 14 genera and 11 families, and the liverworts for eight genera and four families. Rectolejeunea pililoba (Spruce) Schust. is cited for the first time from Brazil. Altogether, 26 species are cited for the first time for the State.

Key words: Hepaticopsida, Bryopsida, Itabaiana.

\section{Introdução}

A Estação Ecológica da Serra de Itabaiana (1043-46’S, 37¹8-23'W), está localizada nos municípios de Areia Branca e Itabaiana no estado de Sergipe. Tem uma área de 288,53 hectares já regularizados como Estação e ainda ca. 1100 hectares para serem regularizados. Esta área é conhecida como Serra de Itabaiana e faz parte de um complexo denominado Serra Residual, com cotas altimétricas variando entre 200$860 \mathrm{~m}$.

A região apresenta diversidade de formações vegetais como campo rupestre, campos, restinga, cerrado e remanescente de Mata Atlântica recortados por recursos hídricos.

Para a região haviam sido referidas apenas algumas espécies de briófitas como: Sphagnum palustre L. (Yano et al. 1985), Frullanoides tristis (Steph.) Van Slageren (Van Slageren 1985), Frullania neesii Lindenb. (Yano et al. 1987), Riccia vitalii

\footnotetext{
${ }^{1}$ Instituto de Botânica, Caixa Postal 4005, 01061-970, São Paulo-SP, Brasil.
} 
Jovet-Ast (Jovet-Ast 1987), Orthostichopsis tetragona (Sw. ex Hedw.) Broth., Sematophyllum subsimplex (Hedw.) Mitt., Trichosteleum papillosum (Hornsch.) Jaeg. (Yano 1992a) e Octoblepharum albidum Hedw. (Marinho 1987, Yano 1992b).

O objetivo do trabalho é estudar as briófitas desta região auxiliando na distribuição geográfica das espécies para o Brasil.

\section{Material e métodos}

Exame dos materiais existentes no Herbário do Estado "Maria Eneyda P. Kauffmann Fidalgo" (SP) e levantamento em literatura das espécies já citadas para a região de Serra Itabaiana, Sergipe.

Faz-se um pequeno comentário do substrato e dos caracteres mais importantes para a identificação e se menciona a distribuição geográfica brasileira para cada espécie.

As amostras foram identificadas com base em Bartram (1949), Arnell (1963), Schuster (1980), Buck (1983), Buck \& Ireland (1985) e comparadas com os espécimes já identificados por especialistas.

A classificação das espécies de Hepaticopsida segue a de Schuster (1984) e Bryopsida de Vitt (1984) com algumas modificações.

\section{Resultados e comentários específicos}

Hepaticopsida

Lepidoziaceae

Zoopsis integrifolia (Spruce) Steph., Spec. Hepat. 3: 284. 1908.

Basiônimo: Cephalozia integrifolia Spruce, J. Bot. London 14: 136. 1876.

Tipo: Brasil, Amazonas, Uaupés.

Material examinado - Sergipe, município de Itabaiana-Areia Branca, Estação Ecológica da Serra de Itabaiana, na margem do riacho perto do campo rupestre, col. O. Yano, M.P. Marcelli \& M. Costa 16504, 24-I-1992 (SP 241655).

Comentários - Cresce no solo úmido sobre faces úmidas de rochedos e em troncos de árvores caídas em florestas bem úmidas.

A espécie tem os filídios geralmente ovalado-assimétricos, de ápice largo, arredondado e é dióica.

Ocorre nos estados do Amazonas, Mato Grosso e São Paulo.

Geocalycaceae

Lophocolea martiana Nees in G.L. \& N., Syn. Hepat. 152. 1845.

Tipo: Brasil. 
Material examinado - Sergipe, município de Itabaiana-Areia Branca, Estação Ecológica da Serra de Itabaiana, no solo arenoso e pedra perto do riacho, col. O. Yano, M.P. Marcelli \& M. Costa 16518, 16523, 24-I-1992 (SP 241668, SP 241673).

Comentários - Cresce no solo úmido e madeira em decomposição nas matas e riachos.

A espécie apresenta a margem superior do filídio inteira, sem dentes.

Ocorre nos estados do Amazonas, Amapá, Espírito Santo, Minas Gerais, Pará, Paraná, Pernambuco, Rio de Janeiro, Rio Grande do Sul, Santa Catarina e São Paulo.

Frullaniaceae

Frullania caroliniana Sull., Musci Alleghan. 64. 1846.

Tipo: USA, Carolina do Norte, perto de Wilmington.

Material examinado - Sergipe, município de Itabaiana-Areia Branca, Estação Ecológica da Serra de Itabaiana, na base de arbusto da restinga, col. O. Yano, M.P. Marcelli \& M. Costa 16542, 24-I-1992 (SP 241690).

Comentários - Cresce aderida à casca das árvores na beira de matas em locais abertos e iluminados pelo sol.

Nesta espécie ocorrem com freqüência ramos laterais situados logo abaixo do perianto parecendo à primeira vista uma inovação.

Ocorre nos estados do Espírito Santo, Rio Grande do Sul e São Paulo.

Frullania dusenii Steph. in Dusén, Archos Mus. Nat. Rio de Janeiro 13: 115. 1903. Tipo: Brasil, Rio de Janeiro, Serra de Itatiaia, Retiro dos Ramos.

Material examinado - Sergipe, município de Itabaiana-Areia Branca, Estação Ecológica da Serra de Itabaiana, na base do arbusto na restinga, col. O. Yano, M.P. Marcelli \& M. Costa 16493, 16545, 24-I-1992 (SP 241645, SP 241692).

Comentários - Foi encontrada na base de arbusto na restinga.

Esta espécie pode ser caracterizada por possuir os filídios com lóbulos sacados, arredondados, inflados especialmente na porção superior e distal, os quais se ligam ao lobo através de uma dobra bem curta. Os anfigastros são ca. 3 vezes mais largos que o caulídio e brevemente bífidos. Quando fértil o perianto se liga às duas brácteas laterais.

Ocorre nos estados do Rio de Janeiro, Rio Grande do Sul, Santa Catarina e São Paulo.

Frullania ecklonii (Spreng.) Spreng. in Gott., Lindenb. \& Nees, Syn. Hepat. 413. 1845.

Basiônimo: Jungermannia ecklonii Spreng., L. f., Syst. Veg. 4(2): 324. 1827.

Tipo: África do Sul

Material examinado - Sergipe, município de Itabaiana, rio Tabora, "growing on shrub trunks in low and sparse forest (caatinga), along a temporary river", col. D.M. Vital 2870, 29-I-1974, det. S. Hattori (SP 88376). 
Comentários - Cresce sobre raízes de árvores em matas bem abertas ou próximo de rios ou cachoeiras.

A espécie tem o anfigastro quase arredondado com uma pequena depressão no ápice; o lóbulo largo, paralelo ao caulídio, curto claviforme na porção superior.

Esta espécie é próxima de $F$. affinis Nees segundo Arnell (1963).

Ocorre no Rio de Janeiro (Herbário de Hooker; Gottsche et al. 1844); Serra de Itatiaia (RJ) e São Paulo (Yuzawa 1991).

Frullania ericoides (Nees) Nees, Syn. Hepat. 417. 1845.

Basiônimo: Jungermannia ericoides Nees in Mart., Flora Bras. enum. plant. 1(1): 346. 1833.

Tipo: Brasil, Rio de Janeiro.

Material examinado - Sergipe, Itabaiana, Areia Branca, col. E.C. Oliveira Filho \& Moacir s/n, 5-XII-1974 (SP 148201p.p.); idem, Estação Ecológica da Serra de Itabaiana, na base do arbusto na restinga, col. O Yano, M.P. Marcelli \& M. Costa 16497, 24-I-1992 (SP 242648).

Comentários - Cresce geralmente sobre troncos de árvores de casca lisa de vegetação bem aberta ou de árvores isoladas.

A espécie pode ser reconhecida pelos filídios fortemente esquarrosos e pelos periantos 3-quilhados e com ornamentação na base.

Ocorre nos estados de Bahia, Espírito Santo, Goiás, Minas Gerais, Mato Grosso, Pernambuco (Fernando de Noronha e continente), Rio de Janeiro, Rio Grande do Sul e São Paulo.

Frullania gymnotis Nees \& Mont., Ann. Sci. Nat. Paris, sér. 2. 19: 257. 1843.

Tipo: Guiana.

Material examinado - Sergipe, município de Itabaiana-Areia Branca, Estação Ecológica da Serra de Itabaiana, sobre pedra no campo rupestre, col. O. Yano, M.P. Marcelli \& M. Costa 16517, 16533, 24-I-1992 (SP 241667, SP 241683); idem, na base do arbusto na restinga, col. O. Yano, M.P. Marcelli \& M. Costa 16544, 16560, 16563, 24-I-1992 (SP 241691, SP 241703, SP 241706).

Comentários - Cresce sobre pedra no campo ou muito aderido ao tronco geralmente na base em locais abertos.

Pode ser distinta de Frullania neesii Lindenb. porque possui os lóbulos dos filídios sacados e inclinados, mas com a porção superior mais afastada do caulídio formando com este um ângulo de aproximadamente $30^{\circ} \mathrm{e}$ as brácteas e bractéolas periqueciais possuem as margens denteadas.

Ocorre nos estados de Pará, Rio Grande do Sul e São Paulo.

Frullania neesii Lindenb., Syn. Hepat. 450. 1845.

Tipo: Brasil, Minas Gerais, Ouro Preto como Vila Rica.

Material examinado - Sergipe, município de Itabaiana-Areia Branca, Estação Ecológica da Serra de Itabaiana, na base de arbusto na restinga, col. O. Yano, M.P. Marcelli 
\& M. Costa 16490, 16492, 16496, 16503, 16539, 16549, 16564, 24-I-1992 (SP 241642, SP 241644, SP 241648, SP 241654, SP 241687, SP 241696, SP 241707); idem, sobre tronco de Vellozia na restinga, col. O. Yano, M.P. Marcelli \& M. Costa 16559, 24-I-1992 (SP 241702).

Comentários - Cresce aderida ao tronco das árvores em locais abertos.

A espécie possui os lóbulos dos filídios sacados e subparalelos ao caulídio ou inclinados com a porção superior mais próxima ao caulídio do que a base. Brácteas e bractéolas com as margens lisas. Muito semelhante a $F$. gymnotis, que tem os lóbulos subparalelos ao caulídio e a porção superior afastada do mesmo, além das brácteas e bractéolas com as margens denteadas.

Ocorre nos estados do Ceará, Espírito Santo, Sergipe, Minas Gerais, Mato Grosso, Pernambuco, São Paulo e Rio Grande do Sul.

Frullania riojaneirensis (Raddi) Spruce, Trans. Proc. Bot. Soc. Edinburg 15: 23. 1884.

Basiônimo: Frullanoides riojaneirensis Raddi, Atti. Sci. Ital. Sci. Modena 19: 37. 1822.

Tipo: Brasil, Rio de Janeiro, Monte Corcovado.

Material examinado - Sergipe, município de Itabaiana-Areia Branca, Estação Ecológica da Serra de Itabaiana, na base do arbusto na restinga, col. O. Yano, M.P. Marcelli \& M. Costa 16499, 24-I-1992 (SP 241651).

Comentários - Cresce na base dos troncos de arbusto na restinga bem aberta e iluminada.

, Distingue-se pelos lóbulos do filídio grandes, inflados na porção superior e com o segmento laminar basal bem desenvolvido e pelos anfigastros várias vezes mais largos que o caulídio, brevemente bífidos no ápice e auriculados na base; as brácteas e bractéolas têm margens lisas e o perianto é trapezoidal em seção transversal.

Ocorre nos estados da Bahia, Distrito Federal, Espírito Santo, Goiás, Mato Grosso, Minas Gerais, Pernambuco, Rio de Janeiro, Rio Grande do Sul e São Paulo.

\section{Lejeuneaceae}

Bryopteris diffusa (Sw.) Nees in Gott., Lindenb. \& Nees, Syn. Hepat. 286. 1845. Basiônimo: Jungermannia diffusa Sw., Flora Ind. Occid. Prod. 144. 1788. Tipo: Jamaica.

Material examinado - Sergipe, Itabaiana, Areia Branca, col. E.C. Oliveira Filho \& Moacir s/n, 5-XII-1974 (SP 148201p.p.); idem, Estação Ecológica da Serra de Itabaiana, na base de arbusto na restinga, col. O. Yano, M.P. Marcelli \& M. Costa 16546, 24-I-1992 (SP 241693).

Comentários - Cresce sobre galhos e troncos de árvores de matas úmidas e áreas não poluídas.

A espécie pode ser distingüida pelos ramos com aparência dicotômica e a margem dos lóbulos livres com 3 dentes. 
Ocorre nos estados do Alagoas, Amazonas, Bahia, Espírito Santo, Mato Grosso, Minas Gerais, Pará, Paraná, Pernambuco, Rio Grande do Sul, Rio de Janeiro, Santa Catarina e São Paulo.

Diplasiolejeunea rudolphiana Steph., Hedwigia 35: 79. 1896.

Tipo: Brasil, Rio de Janeiro, Petrópolis.

Material examinado - Sergipe, município de Itabaiana-Areia Branca, Estação Ecológica da Serra de Itabaiana, na base de arbusto na restinga, col. O. Yano, M.P. Marcelli \& M. Costa 16501, 24-I-1992 (SP 241653).

Comentários - Cresce sobre troncos de árvores e arbustos em locais bem iluminados.

A espécie apresenta um anfigastro peculiar lobado-divaricado e no lóbulo um dente apical acuminado, delgado e proeminente. Ainda pode ser distinta pelos ocelos laminares esparsos nas células do lobo dorsal.

Ocorre nos estados do Espírito Santo, Pernambuco, Rio de Janeiro e São Paulo.

Microlejeunea ulicina (Tayl.) Steph., Hedwigia 29: 88. 1890.

Basiônimo: Jungermannia ulicina Tayl., Trans. Bot. Soc. Edinburg 1: 115. 1844.

Tipo: Irlanda.

Material examinado - Sergipe, município de Itabaiana, rio Tabora, growing on base of a shrub trunk, in a low and sparse forest (caatinga), along a temporary river, col. D.M. Vital 2871p.p. 29-I-1974 (SP 88377).

Comentários - Cresce primariamente como pioneira em substratos (rochas, troncos) de superfície lisa.

A espécie possui o lóbulo ocupando 2/3 do filídio; são plantas muito pequenas.

Ocorre nos estados do Espírito Santo e Roraima.

Rectolejeunea pililoba (Spruce) Schust., The Hepaticae and Anthocerotae of North America 4: 1153. 1980.

Basiônimo: Lejeunea pililoba Spruce, J. Linn. Soc. Bot. 30: 346. 1895.

Tipo: Dominica.

Material examinado - Sergipe, município de Itabaiana, rio Tabora, "growing on base of a shrub, in a low and sparse forest (caatinga), along temporary river", col. D.M. Vital 2868, 29-I-1974 (SP 88374).

Comentários - Cresce na base dos troncos de árvores, sobre Leptogium cf. cyanescens (Rabenh.) Koerber (líquen). Segundo Schuster (1980) é estritamente corticícola.

O lóbulo do filídio é uniformemente reduzido a poucas células, filiforme com (2-3)4-12 células de comprimento, unisseriado no ápice e 2-3 células de largura na base, com papila terminal hialina.

Está sendo citada pela primeira vez para o Brasil, sendo que na América do Sul ocorre da Guiana à Venezuela.

Schiffneriolejeunea polycarpa (Nees) Gradst., J. Hattori Bot. Lab. 38: 335. 1974. Basiônimo: Jungermannia polycarpa Nees in Mart., Flora Bras. enum. plant. 1(1): 
350. 1833.

Tipo: Brasil, Minas Gerais.

Material examinado - Sergipe, município de Itabaiana-Areia Branca, Estação Ecológica da Serra de Itabaiana, sobre tronco e base de árvore na restinga, col. O. Yano, M.P. Marcelli \& M. Costa 16494, 16498, 24-I-1992 (SP 241646, SP 241650).

Comentários - Cresce aderida ao substrato principalmente sobre caule de arbusto ou árvore.

Pode ser reconhecida pelos oleocorpos segmentados como em Mastigolejeunea, ápice da bractéola bífido, lóbulo do filídio curto, ligeiramente mais longo que alto.

Ocorre nos estados do Amazonas, Espírito Santo, Minas Gerais, Pará, Pernambuco, Rio de Janeiro, Rio Grande do Sul e São Paulo.

Bryopsida

Orthotrichaceae

Groutiella schlumbergeri (Schimp.) Wijk \& Marg., Taxon 9: 51. 1960.

Basiônimo: Micromitrium schlumbergeri Schimp. in Besch., Mem. Soc. Sc. Nat. Cherbourg 16: 191. 1872.

Tipo: ?

Material examinado - Sergipe, município de Itabaiana, rio Tabora, "growing $2 \mathrm{~m}$ up on a shrubby tree trunk, on the banks of a temporary river", col. D.M. Vital 2874, 29-I1974, det. D. Vital (SP 90784); idem, bordo da mata ciliar, na base de troncos, col. E.C. Oliveira Filho \& Moacir s/n, 5-XII-1974 (SP 148197).

Comentários - Cresce sobre troncos de árvores e nos barrancos de rio temporário.

Yano (1981) se refere a esta espécie como Groutiella fragilis para Amazonas, Mato Grosso, Pará e Pernambuco.

Macromitrium pellucidum Mitt., J. Linn. Soc. Bot. 12: 203. 1869.

Tipo: Brasil, Amazonas.

Material examinado - Sergipe, município de Itabaiana, Serra de Itabaiana, em tronco de árvore viva, col. D. Andrade-Lima 81-6741, 13-I-1981 (SP 171164).

Comentários - Cresce sobre troncos de árvores formando tapete denso em matas abertas ou árvores isoladas.

A espécie pode ser reconhecida pelas células alongadas de paredes bem espessadas e ápice do filídio mucronado; a caliptra cobrindo toda cápsula, o que não é normal no gênero.

Ocorre nos estados do Amazonas e Mato Grosso.

Bryaceae

Bryum sub-verticillatum (Broth.) Ochi, J. Fac. Educ. Tottori Univ. 30: 29. 1981. 
Basiônimo: Rhodobryum subverticillatum Broth., Denkschr. Akad. Wiss. Wien. Math. Nat. Kl. 83: 299. 1926.

Tipo: Brasil, São Paulo, Lapa, Schiffner 763.

Material examinado - Sergipe, Itabaiana, Areia Branca, sobre o solo, col. E.C. Oliveira Filho \& M. Fonseca s/n, 5-XII-1974 (SP 148203); idem, sobre "litter" (SP 148204); idem, "growing on ground in a low (3m tall) and sparse forest (caatinga), along a temporary river”, col. D.M. Vital 2872, 29-I-1974 (SP 90792); idem, Estação Ecológica da Serra de Itabaiana, nas frestas da rocha no campo rupestre, col. O. Yano, M.P. Marcelli \& M. Costa 16534, 24-I-1992 (SP 241684).

Comentários - Cresce no solo ou sobre húmus de matas mais abertas.

Esta espécie se assemelha muito a Bryum beyrichianum (Hornsch.) C. Muell., mas pode ser diferenciada pelo bordo do filídio indistinto com 3-4 fileiras de células estreitas e alongadas, o que não é encontrado em $B$. beyrichianum; os filídios são oblongo-espatulados e a base pouco estreita.

Ocorre nos estados de Minas Gerais, Santa Catarina e São Paulo.

Plagiotheciaceae

Eulacophyllum cultelliforme (Sull.) Buck \& Ireland, Nova Hedwigia 41: 108. 1985. Basiônimo: Hypnum cultelliforme Sull., Proc. Amer. Acad. Arts 5: 289. 1861.

Tipo: Cuba.

Material examinado - Sergipe, Itabaiana, Areia Branca, col. E.C. Oliveira Filho \& Moacir s/n, 5-XII-1974 (SP 148200p.p.).

Comentários - Cresce sobre troncos de árvores. São plantas muito pequenas.

A espécie monotípica se caracteriza pelas células do filídio com brusco espessamento terminal dando impressão de papilas e pelas células alares quadráticas a retangulares pequenas, terminando junto da costa; a margem é denteada na metade superior.

Ocorre apenas nos estados de Espírito Santo e Paraíba.

Fabroniaceae

Fabronia ciliaris (Brid.) Brid. var. polycarpa (Hook.) Buck, Brittonia 35(3): 251. 1983.

Basiônimo: Fabronia polycarpa Hook., Musci Exot. 1: 3. 1818.

Tipo: Colômbia.

Material examinado - Sergipe, Itabaiana, Areia Branca, col. E.C. Oliveira Filho \& Moacir s/n, 5-XII-1974 (SP 148200p.p.).

Comentários - Cresce sobre troncos de árvores geralmente isoladas das matas ou nos parques e jardins.

A espécie possui os filídios mais ou menos ovalados, ápice acuminado e margem tipicamente inteira; células com paredes delgadas.

Ocorre nos estados da Bahia, Ceará, Espírito Santo, Minas Gerais, Paraná, 
Pernambuco, Rio Grande do Sul, Rio de Janeiro, Santa Catarina e São Paulo.

Sematophyllaceae

Sematophyllum subsimplex (Hedw.) Mitt., J. Linn. Soc. Bot. 12: 494. 1869.

Basiônimo: Hypnum subsimplex Hedw., Spec. Musc. 270. 1801.

Tipo: Ilhas Ocidentais.

Material examinado - Sergipe, município de Itabaiana-Areia Branca, Estação Ecológica da Serra de Itabaiana, no solo úmido perto do riacho, col. O. Yano, M.P. Marcelli \& M. Costa 16520, 24-I-1992 (SP 241670).

Comentários - Cresce geralmente sobre madeira podre na mata ou nas margens de regatos ou riachos.

Pode ser facilmente reconhecida pelo sistema de ramificação subpinado e de ramos horizontais, pelo filídio oblongo e estreitamente acuminado, células alares infladas na base e células da lâmina muito alongadas, sinuosas e lisas.

Ocorre nos estados do Amazonas, Amapá, Distrito Federal, Espírito Santo, Goiás, Maranhão, Mato Grosso, Minas Gerais, Pará, Paraíba, Paraná, Pernambuco, Rio de Janeiro, Rio Grande do Sul, Roraima, Santa Catarina, São Paulo e Sergipe.

\section{Meteoriaceae}

Squamidium gracilescens (Broth.) Broth., Naturl. Pflanzenfam. 1(3): 809. 1906. Basiônimo: Pilotrichella gracilescens Broth., Bih. K. Svensk. VetenskAkad. Handl. 3(7): 41. 1900.

Tipo: Brasil, Rio Grande do Sul.

Material examinado - Sergipe, município de Itabaiana, Serra de Itabaiana, ca. 350m alt., em tronco de árvore viva, col. D. Andrade-Lima 81-6742, 13-I-1981 (SP 171165).

Comentários - Cresce sobre ramos e troncos de árvores na mata aberta.

Os ramos são afilados; os filídos dos ramos pouco mais estreitos com longa ponta filiforme.

Ocorre nos estados do Paraná, Pernambuco e Rio Grande do Sul.

\section{Callicostaceae}

Schizomitrium pallidum (Hornsch.) Crum \& Anderson, Mosses E. N. Amer. 2: 822. 1981.

Basiônimo: Hookeria pallida Hornsch. in Mart., Flora Bras. 1(2): 64. 1840.

Tipo: Brasil, Minas Gerais.

Material examinado - Sergipe, município de Itabaiana-Areia Branca, Estação Ecológica da Serra de Itabaiana, sobre pedra no leito do riacho na entrada da estação, col. O. Yano, M.P. Marcelli \& M. Costa 16568, 24-I-1992 (SP 241711).

Comentários - Cresce sobre tronco em decomposição, margem de riachos ou no leito 
dos rios periodicamente inundados.

Caracteristicamente, os filídios não são bordeados por células pequenas e isodiamétricas; a costa é dupla e as células da metade superior dos filídios têm uma papila na superfície dorsal.

Ocorre nos estados do Amazonas, Amapá, Espírito Santo, Goiás, Mato Grosso, Minas Gerais, Pará, Paraná, Pernambuco, Rio de Janeiro, Rio Grande do Sul, Roraima e São Paulo.

\section{Pottiaceae}

Weisia termitarum C. Muell., Hedwigia 39: 267. 1900.

Tipo: Brasil, Goiás, Moçamedes.

Material examinado - Sergipe, município de Itabaiana, rio Tabora, growing in partial shade, on gullied ground, along a temporary river, col. D.M. Vital 2873, 29-I-1974, det. R. Zander (SP 90793).

Comentários - Cresce sobre o solo ao longo do rio temporário em local parcialmente sombrio.

A amostra foi identificada em 1991 por Zander, especialista em Pottiaceae como pertencente ao gênero Weisia embora o Index Muscorum de Wijk et al. (1969) considere como válido o nome Hymenostomum termitarum (C. Muell.) Broth.

Ocorre apenas no estado de Goiás.

\section{Calymperaceae}

Calymperes palisotii Schwaegr. subsp. richardii (C. Muell.) S. Edwards, J. Bryol. 11: 81. 1980.

Basiônimo: Calymperes richardii C. Muell., Syn. 1: 524. 1849.

Tipo: Brasil, Pernambuco.

Material examinado - Sergipe, município de Itabaiana-Areia Branca, Estação Ecológica da Serra de Itabaiana, sobre tronco de árvore, col. O. Yano, M.P. Marcelli \& M. Costa 16486, 24-I-1992 (SP 241638).

Comentários - Cresce geralmente sobre tronco de árvore bem exposta ao sol.

A espécie pode ser facilmente reconhecida pelo ápice obtuso, freqüentemente com um tufo de gemas; quando fértil tem a caliptra paleácea, sulcada, recobrindo toda a cápsula; as teníolas geralmente pouco evidentes.

Ocorre nos estados de Alagoas, Amazonas, Amapá, Bahia, Espírito Santo, Fernando de Noronha, Goiás, Paraná, Paraíba, Pernambuco, Rio de Janeiro, Rondônia e Roraima.

Syrrhopodon prolifer Schwaegr., Spec. Musc. Suppl. 2(2): 99. 180. 1827.

Tipo: Brasil.

Material examinado - Sergipe, Itabaiana, Areia Branca, col. E.C. Oliveira Filho \& Moacir s/n, 5-XII-1974 (SP 148201p.p., SP 148202); idem, Estação Ecológica da 
Serra de Itabaiana, na margem do riacho perto do campo rupestre, col. O. Yano, M.P. Marcelli \& M. Costa 16509, 24-I-1992 (SP 241659).

Comentários - Cresce sobre troncos de árvores e rochas de locais relativamente altos em florestas pluviais.

Esta espécie pode ser distinta pelo ápice do filídio agudo, as margens conspícuas e as células superiores obscuras e pluripapilosas.

Ocorre nos estados da Bahia, Goiás, Minas Gerais, Pará, Pernambuco, Rio Grande do Sul, Rio de Janeiro, Roraima, Santa Catarina e São Paulo.

\section{Dicranaceae}

Campylopus arenaceum (Broth.) J.P. Frahm, J. Bryol. 8(2): 258. 1974.

Basiônimo: Thysanomitrium arenaceum Broth., Denkschr. Akad. Wiss. Wien. math. nat. Kl. 83: 264. 1926.

Tipo: Brasil, São Paulo.

Material examinado - Sergipe, município de Itabaiana, Serra de Itabaiana, no solo arenoso, col. D. Andrade-Lima 81-6826, 13-I-1981 (SP 171169); idem, Estação Ecológica da Serra de Itabaiana, no solo arenoso branco da restinga, col. O. Yano, M.P. Marcelli \& M. Costa, 24-I-1992 (SP 242641).

Comentários - Cresce no solo arenoso perto da vegetação mais aberta.

Esta espécie pode ser reconhecida pelas células alares arredondadas em 4-5 fileiras e as células superiores do filídio muito pequenas e mais largas do que longas; ápice do filídio hialino com dentes bem evidentes.

Ocorre nos estados de Espírito Santo, Goiás e São Paulo.

\section{Leucobryaceae}

Leucobryum martianum (Hornsch.) Hampe, Linnaea 17: 317. 1843.

Basiônimo: Dicranum martianum Hornsch. in Mart., Flora Bras. 1(2): 11. 1840.

Tipo: Brasil, Minas Gerais, Caldas.

Material examinado - Sergipe, município de Itabaiana-Areia Branca, Estação Ecológica da Serra de Itabaiana, na margem do riacho, col. O. Yano, M.P. Marcelli \& M. Costa 16514, 24-I-1992 (SP 241664).

Comentários - Cresce geralmente sobre troncos podres em solo arenoso nas proximidades de água.

A espécie é caracterizada pelos filídios falcados unilateralmente e no corte transversal do filídio, a camada clorocística está mais perto da superfície ventral. As células leucocísticas do lado dorsal são convexas ao longo de todo o filídio.

Ocorre nos estados do Acre, Alagoas, Amazonas, Amapá, Ceará, Distrito Federal, Espírito Santo, Maranhão, Minas Gerais, Pará, Paraná, Pernambuco, Rio de Janeiro, Rondônia, Roraima, Santa Catarina e São Paulo. 
Tipo: Jamaica.

Material examinado - Sergipe, Itabaiana, Rio das Mortes, sobre areia em vegetação de restinga, col. E.C. Oliveira Filho \& M. Fonseca s/n, 5-XII-1974, det. D. Vital (SP 148198); município de Itabaiana, Serra de Itabaiana, + 350m alt. em tronco de árvore podre, col. D. Andrade-Lima 81-6743, 13-I-1981 (SP 171166); idem, Estação Ecológica da Serra de Itabaiana, na base do arbusto no campo rupestre, col. O. Yano, M.P. Marcelli \& M. Costa 16556, 24-I-1992 (SP 241699); idem, na restinga, col. O. Yano, M.P. Marcelli \& M. Costa 16557, 24-I-1992 (SP 241700).

Comentários - Cresce sobre troncos de árvores ou na base, sobre pedras de matas mais abertas.

Quando estéril pode ser identificado pelo ápice do filídio com pequenos dentículos.

Ocorre em todos os estados brasileiros, menos em Tocantins.

Octoblepharum albidum Hedw. var. violascens C. Muell., Linnaea 19: 208. 1846. Tipo: Colômbia, Caripe.

Material examinado - Sergipe, município de Itabaiana-Areia Branca, Estação Ecológica da Serra de Itabaiana, na base do arbusto na restinga, col. O. Yano, M.P. Marcelli \& M. Costa 16488, 24-I-1992 (SP 241640); idem, sobre tronco de arbusto perto do riacho, ca. 220m alt., col. O. Yano, M.P. Marcelli \& M. Costa 16527, 24-I-1992 (SP 241677).

Comentários - Cresce geralmente sobre troncos podres nas matas.

A variedade violascens $\mathrm{C}$. Muell. difere da variedade albidum por ter filídios mais largos, delicados e com manchas purpúreas ou violáceas na base.

Ocorre nos estados do Acre, Amazonas, Mato Grosso, Pará, Rio de Janeiro, Rondônia e São Paulo.

Octoblepharum cocuiense Mitt., J. Linn. Soc. Bot. 12: 109. 1869.

Tipo: Colômbia, Monte Cucui.

Material examinado - Sergipe, município de Itabaiana-Areia Branca, Estação Ecológica da Serra de Itabaiana, na margem do riacho, col. O. Yano, M.P. Marcelli \& M. Costa 16513, 24-I-1992 (SP 241663); idem, sobre pedra perto do riacho, col. O. Yano, M.P. Marcelli \& M. Costa 16531, 24-I-1992 (SP 241681).

Comentários - Cresce na sombra, sobre pedras e rochas próximos a rios ou riachos que recebem respingos de quedas de água, ou ainda nas frestas de rochas em locais mais secos.

Esta espécie apresenta geralmente coloração rósea a purpúrea, são bastante quebradiças. O ápice do filídio é agudo ou levemente acuminado, às vezes apiculado, levemente ondulado, as aletas são desiguais, sendo uma bem maior que a outra e as células da lâmina sem pontuações na parede.

Ocorre nos estados do Acre, Amazonas, Bahia, Espírito Santo, Goiás, Mato Grosso, Minas Gerais, Paraná, Rio de Janeiro, Rondônia e São Paulo. 


\section{Referências bibliográficas}

Arnell, S. 1963. Hepaticae of South Africa. Sweden: A. Norstedt \& Söner. 411 p.

Bartram, E.B. 1949. Mosses of Guatemala. Fieldiana, Bot. 25: 1-442.

Buck, W.R. 1983. A synopsis of the South American taxa of Fabronia (Fabroniaceae). Brittonia 35(3): 248-254.

Buck, W.R. \& Ireland, R.R. 1985. A reclassification of the Plagiotheciaceae. Nova Hedwigia 41: 89-125.

Gottsche, C.M., Lindenberg, I.B.G. \& Nees, C.G. 1844. Synopsis Hepaticarum, Hamburgii. 835p.

Jovet-Ast, S. 1987. Un Riccia nouveau du Nord-est du Brésil: Riccia vitalii (Ricciaceae, sous-genre Riccia). Mem. N.Y. Bot. Gdn 45: 283-288.

Marinho, M.G.V. 1987. Bryopsida na reserva florestal do IBDF, João Pessoa, Paraíba, Brasil. Recife: Univiversidade Federal de Pernambuco. Dissertação de Mestrado.

Schuster, R.M. 1980. The Hepaticae and Anthocerotae of North America east of the hundredth Meridian. 4, New York: Columbia Univ. Press.

Schuster, R.M. 1984. Evolution, phylogeny and classification of the Hepaticae. In: Schuster, R. M. (ed.). New Manual of Bryology: Tokyo: Hattori Botanical Laboratory, 2: 892-1070.

Van Slageren, M.W. 1985. A taxonomic monograph of the Brachiolejeunea and Frullanoides (Hepaticae) with a SEM analysis of the sporophyte in the Ptychanthoideae. Netherlands.

Vitt, D.H. 1984. Classification of the Bryopsida. In: Schuster, R.M. (ed.). New Manual of Bryology: Tokyo: Hattori Botanical Laboratory, 2: 696-759.

Wijk, R. van, Margadant, W.D. \& Florschütz, P.A. 1969. Index Muscorum 5: v-xii + 1-922.

Yano, O. 1981. A checklist of Brazilian mosses. J. Hattori Bot. Lab. 50: 279-456.

Yano, O. 1992a. Novas localidades de musgos nos estados do Brasil. Acta Amazonica 22(2): 197-218.

Yano, O. 1992b. Leucobryaceae (Bryopsida) do Brasil. São Paulo: Universidade de São Paulo. Tese do Doutorado.

Yano, O.; Pirani, J.R. \& Santos, D.P. 1985. O Gênero Sphagnum (Bryopsida) nas regiões Sul e Sudeste do Brasil. Revta brasil. Bot. 8(1): 55-80.

Yano, O.; Marinho, M.G.V. \& Mariz, G. 1987. Novas ocorrências de briófitas no Nordeste Brasileiro. Rickia 14: 73-87.

Yuzawa, Y. 1991. A monograph of subg. Chonanthelia of gen. Frullania (Hepaticae) of the world. $J$. Hattori Bot. Lab. 70: 181-291. 